\title{
An Investigation and Analysis of the Relationship between College Students' Information Anxiety and Coping Style under Internet Background
}

\author{
Ameng Zhao ${ }^{1, a}$, Gang Chen ${ }^{1, b}$, Panpan Yang ${ }^{1, c},{ }^{1,}$ uhua Wang ${ }^{1, d}$, \\ Zhenzhen Cai ${ }^{1, \mathrm{e}}$, Jinling $\mathrm{Qi}^{1, \mathrm{f}}$, Xiaoyu Zhen ${ }^{1, \mathrm{~g}}$ \\ ${ }^{1}$ Qiqihar Medical University, Qiqihar, 161006, China \\ a113348606@qq.com, bfanchuanlo@qq.com, c1823777172@qq.com, d69724849@qq.com, \\ e253439565@qq.com, ${ }^{\mathrm{e}} 113357706 @ q q . c o m,{ }^{9} 396171469 @ q q . c o m$
}

Keywords:Internet;College students;Information anxiety;Coping style

\begin{abstract}
To explore the relationship between college students' information anxiety and coping style under the background of Internet,and to provide a reference for college student's mental health. In March 2017 a total of 1010 college students were measured with Information Anxiety Scale and Adolescent Coping Style Assessment Questionnaire.There was no significant difference in information anxiety among college students of different genders.( $\mathrm{t}=1.646, \mathrm{p}>0.05)$. Male college students tend to use problem-solving $(\mathrm{t}=2.767, \mathrm{p}<0.05)$ and fantasy coping styles $(\mathrm{t}=2.192, \mathrm{p}<$ 0.05);high anxiety group of college students tend to use the problem-solving coping style $(\mathrm{t}=2.405, \mathrm{p}$ $<0.05)$, while fewer use of fantasy coping styles $(\mathrm{t}=4.965$,p $<0.05)$. Multiple linear regression analysis showed that problem-solving and fantasy coping styles have predictive effects on college students' information anxiety. The coping style of college students can predict the information anxiety mood.Colleges and universities should be targeted to carry out the corresponding education and advocacy work.
\end{abstract}

\section{Introduction}

With the rapid development of the social economy, especially the rapid spread of network information, it has produced a huge impact on the modern way of life and thinking habits and mentality. As the most influential college student group in the information society, it is particularly affected by the Internet [1]. At present, the informational anxiety has become the more common negative emotions of college students. Although anxiety has some positive meanings, if the students are anxious for a long time, Their psychosomatic damage to a certain extent [2]. The relevant research shows that inappropriate coping styles will have a great impact on anxiety [3]. Information anxiety is the result of social development and progress, but how to deal with this kind of anxiety change effectively as an individual in development, and science arouse their own behavior are important issues concerning the healthy development of students. In order to understand whether there is a difference in the impact of different coping styles on college students' information anxiety, this study conducted a questionnaire survey on 1010 college students in March 2017 in our city and is reported as follows.

\section{Objects and methods}

\subsection{Objects}

Using cluster sampling method, 15 classes were selected from Qiqihar Medical University and Qiqihar University, a total of 1120 subjects, distributed 1120 questionnaires, 1010 valid questionnaires were collected, the recovery efficiency was $90.18 \%$. Among them, there are 490 boys and 520 girls, 190 first graders, the second graders 190, the third graders 360 and the fourth graders 
270.

\subsection{Research tools}

\subsubsection{Adolescent Coping Style Assessment Questionnaire}

Adolescent Coping Style Assessment Questionnaire was prepared by Huang Xiting , a total of 30 items, including six dimensions of the way to help, retreat, vent, fantasy, patience and other coping styles, the higher the score, indicating that the respondents are better at using the dimension of coping the way. The questionnaire using five points, the relevant research shows that the questionnaire has good reliability and validity.

\subsubsection{Information anxiety self-rating scale}

Information anxiety is the result of people's physical and psychological interaction. At present, there is no authoritative information anxiety measurement questionnaire in our country. Therefore, according to the definition of information anxiety, the content of the questionnaire is determined. There are 20 questions and 5 grades in the questionnaire: 100 cases from complete disagreement to complete disagreement. No reverse scoring questions, the higher the score, indicating that the higher the degree of anxiety. In this survey, the questionnaire $\alpha=0.961$

\subsection{Measured program}

After obtaining the consent of the respondents, we conducted a questionnaire survey among classes, class meetings and class activities. The trained task force members took the lead test and adopted standard and uniform directives, asking the respondents to complete the survey independently and anonymously, collected on the spot and further interviewed the students who participated voluntarily. Questionnaire answer time is 10 minutes.

2.4 Statistical methods

Statistical analysis of the data was performed using SPSS17.0. The univariate analysis was performed using t-test. Correlation between the two groups was analyzed by correlation analysis and regression analysis. The difference was statistically significant when $\alpha=0.05$.

\section{Results}

\subsection{Differences in information anxiety among college students of different genders}

In this survey, the male anxiety self-rating scale was $41.82 \pm 9.45$ and the female self-rating anxiety scale was $43.04 \pm 7.23$, the difference was not statistically significant $(\mathrm{t}=1.702, \mathrm{p}>0.05)$

\subsection{Different coping styles of students in different gender situations}

In this survey, students of different genders, apart from boys, preferred to use problem solving and fantasy coping styles. There were no significant differences in the use of coping styles among college students of different genders (Table 1).

Table 1: Differences in coping styles among college students of different genders

\begin{tabular}{lccc}
\hline & Male $(\mathrm{n}=490)$ & Female $(\mathrm{n}=520)$ & $\mathrm{t}$ \\
\hline Problem solved & $20.78 \pm 5.80$ & $17.71 \pm 5.29$ & $2.767^{*}$ \\
Retreat & $10.02 \pm 3.23$ & $9.51 \pm 3.50$ & 0.758 \\
Vent & $6.47 \pm 2.70$ & $7.16 \pm 2.49$ & -1.327 \\
Help & $12.71 \pm 4.86$ & $12.82 \pm 4.88$ & -1.12 \\
Fantasy & $4.02 \pm 2.82$ & $2 \cdot .92 \pm 2.12$ & $2.192^{*}$ \\
Patience & $5.86 \pm 2.35$ & $6.23 \pm 2.04$ & -0.860 \\
\hline
\end{tabular}

Note: ${ }^{*} \mathrm{p}<0.05$

\subsection{The Relationship between college students' information anxiety and coping style}

According to the self-rating anxiety scale of college students, the first $27 \%(n=272)$ and the latter 
$27 \%(n=272)$ were divided into high information anxiety group and low information anxiety group. Differences in coping styles, problem-solving and fantasy coping styles were statistically significant among undergraduates with different levels of information anxiety (Table 2).

Table 2: Differences in coping styles among undergraduates with different levels of information anxiety

\begin{tabular}{lccc}
\hline & $\begin{array}{c}\text { high information } \\
\text { anxiety group }\end{array}$ & $\begin{array}{c}\text { low information } \\
\text { anxiety group }\end{array}$ & $\mathrm{t}$ \\
\hline Problem solved & $20.59 \pm 5.66$ & $17.18 \pm 5.07$ & $2.405^{*}$ \\
Retreat & $9.58 \pm 3.74$ & $9.46 \pm 3.50$ & 0.127 \\
Vent & $6.34 \pm 2.76$ & $7.39 \pm 2.53$ & -1.495 \\
Help & $11.97 \pm 4.81$ & $12.50 \pm 5.08$ & -0.408 \\
Fantasy & $2.03 \pm 2.13$ & $4.89 \pm 2.22$ & $-4.965^{* *}$ \\
Patience & $5.83 \pm 2.17$ & $6.07 \pm 2.52$ & -0.391 \\
\hline
\end{tabular}

Note: ${ }^{*} \mathrm{p}<0.05,{ }^{* *} \mathrm{p}<0.01$

The self-rating anxiety scale was used as the dependent variable, and the six coping styles were scored as independent variables. The multiple linear regression analysis was conducted by using the entering method. The results showed that the fantasy and problem-solving methods entered the self-assessment of college students' information anxiety The regression equation of the scale score. See Table 3 for details.

Table 3: Multiple regression analysis of college students' coping style and anxiety of information

\begin{tabular}{lcccc}
\hline & $\mathrm{B}$ & $\beta$ & $\mathrm{t}$ & Determine the coefficient $\mathrm{R}^{2}$ \\
\hline Fantasy & -1.659 & -0.489 & $-5.789^{* * *}$ & \multirow{2}{*}{0.243} \\
Problem solved & 3.00 & 0.203 & $2.361^{*}$ & \\
\hline
\end{tabular}

Note: ${ }^{*} \mathrm{p}<0.05, * * * \mathrm{p}<0.001$

\section{The discussion}

The concept of information anxiety was first proposed by the American information construction guru Richard Saul Wurman in 1989. It refers to "a black hole between data and knowledge. When the information obtained is not be required "or" tension between the already understood information and the information that should be understood. [4] "With the popularization of information technology, especially the development of the Internet, the massive information makes the individual's information Judgment, information cognition and information processing are all in a more chaotic state. How to deal with and deal with information effectively has become an important content in the personal psychology of modern society. Whether the coping style in traditional psychology research is suitable for this kind of psychological change is a new development of psychology research.

The survey shows that the differences in information anxiety levels among different gender college students are not statistically significant, and some of the conclusions of the study there is a difference [5] , and some of the same conclusions [6]. The reason except for the sample, the survey tools, survey time is different and so on, I believe that with the deepening of the human and the equality of men and women, the fairness of all kinds of information platform, the difference between the traditional social culture and the role of different gender college students is no longer obvious, that is, for college students, relative to gender factors such as college students' self-study and career choices have more significant impact on the level of information anxiety, and are more closely related to specific information anxiety.

The survey shows that boys are more adept at using problem solving and fantasy coping styles than girls. The reason may lie in the fact that the traditional social view holds that boys should be strong and capable, that problems should be solved on their own, and that if they can not be solved It may be used to relieve the pressure by fantasy or other means, instead of using the excuse of exposing their emotions, or by asking others for help. That is, what kind of coping style should be adopted by the individual, apart from being influenced by individual personality traits, it has a greater 
relationship with social culture.

This study shows that undergraduates with high information anxiety are more likely to adopt problem-solving coping styles and less fantasy coping styles, while problem solving and fantasy coping styles have a stronger predictor of information anxiety among undergraduates, The higher the anxiety level of college students who use coping styles, the lower the mental health level, the lower the anxiety level of college students who prefer to use fantasy coping style, the higher the mental health level. Different from the expected "anxiety level of undergraduates using problem solving method". Coping with the problem solving method is indeed conducive to the correct understanding of the problem and improve the predicament, but in the process of solving the problem, it is also bound to face greater pressure to induce information anxiety; from the perspective of psychological defense mechanism, the illusion enables college students in the shortest time solve the problem, but will help maintain the psychological balance, to achieve mental health, but not conducive to the user to solve practical problems [7].

The information anxiety of college students is the result of the interaction of internal personality factors and external environment factors. Therefore, in order to solve the anxiety of undergraduates, we should further enhance the self-regulation ability of undergraduates, and further strengthen the management of network information. We should guide undergraduates to form a correct outlook on life and values, use the internet scientifically, and provide an effective external psychology to undergraduates Support system to help college students better deal with "information anxiety" [8].

\section{Acknowledgement}

In this paper, the research was sponsored by the Heilongjiang Province Philosophy Social Sciences Research Program (No. 16EDB02).

\section{References}

[1] Botao Zeng."Information Anxiety Disorder" of Modern Society [J]. Journal of medical psychology guidance, 2006 (11) : 29-28.

[2] Yanan Zheng,Wen Hu, Yueyue Hu.College Students' Professional Psychological Help Attitude Stigma and Social Support Relationship [J]. Journal of School Health in China, 2016, 5 (1) : 78-80.

[3] Zhuo Chen,Xiuxian Yang,Xiaohui Qiu.Approach to Community Residents Feel Stress and Anxiety Adjustment Effect [J]. Journal of Public Health in China, 2016, 32 (6) : 825-827.

[4] Candace White \& John M. Scheb (2000) . Impact of media messages about the Internet. New media \&society.Vol12 (2): 181- 194.

[5] Richard Joiner, Mark Brosnan (2007), JillDuffield, JeffGavin, PamMaras. The relationship between Internet identification, Internet anxiety and Internet use. Computers in Human Behavior. Vol (23): 1408-1420.

[6] Yaoyang Wei.Study on the Relationship of College Students Anxiety and Information Networks Rely on [J].Intelligence Science, 2014 (11) : 88-91.

[7] Shadiev, R., Hwang, W. Y., Huang, Y. M., \& Liu, T. Y.. The Impact of Supported and Annotated Mobile Learning on Achievement and Cognitive Load[J].Educational Technology \& Society, (2015),18 (4), 53-69.

[8] Flavian C.,Guinaliu M.,Gurrea R.The role played by perceived usability, satisfaction and consumer trust on website loyalty[J].Information \& Management,2006,43(1):1-14. 